\title{
Cloud Computing Platform for Education System: A Review
}

\author{
Yogita Borse \\ Assistant Professor \\ Department of Information Technology \\ $\mathrm{K} \mathrm{J}$ Somaiya College of Engineering \\ Mumbai, Maharashtra 400077, India
}

\author{
Sheetal Gokhale \\ PG Student \\ Department of Information Technology \\ $\mathrm{K} J$ Somaiya College of Engineering \\ Mumbai, Maharashtra 400077, India
}

\begin{abstract}
The education system is about marking and grading system. It is important for students to have practical knowledge, profound thinking, and gain experience and insights different from the traditional or offline education system. As there are pros to offline education, there are cons as well. The offline education system provides a face to face interaction and a better understanding of the world. But apart from the traditional education system, education online is gaining more popularity and attention by many institutions and developers are working better to provide education online at ease. Applications like Byju use Amazon Web Services (AWS) as a cloud computing platform for learning purpose. Online education makes learning process better and conducting practical experience by creating virtual labs for students by scaling up the resources makes it easy for students to have their own hands-on experience using the cloud. Using cloud education saves IT infrastructure cost, and can have ondemand access to the resources needed or required for learning purpose. This paper discusses the cloud architecture implemented by universities using a cloud computing platform for students to have hands-on experience and access to the virtual lab resources that universities fail to provide because of the cost factor. The paper focuses on the private cloud and Infrastructure as a Service (IaaS).
\end{abstract}

\section{Keywords}

Cloud education, Cloud Computing, IaaS, Private cloud, Cloud Architecture.

\section{INTRODUCTION}

Cloud Computing is an emerging trend in the current case scenario. Cloud provides the hardware, software, network interface and other resources virtually on-demand and with pay as you go service benefit. It also helps companies or organizations limit costs on IT infrastructure and maintenance. The data storage location of the application(s) hosted on the cloud from Data Center, within the organization department or by a trusted third party depending upon the Organizations security requirements and perspective. To learn the benefits of cloud and to experience how the cloud operates, many educational institutes came with up with the idea of virtual cloud labs. The primary purpose was to make students aware of Cloud Computing, provide hands-on labs practice with the help of implementing the virtual cloud instance or by using cloud platforms like OpenStack, etc. is discussed in the paper [1][2][3].

Cloud computing resources are provided as virtual services to the customers or group of people with the help of the internet that comprises hardware, software, storage services, data services, and firewall services. The cloud offers key features such as on-demand service, scalability, elasticity, and pas as you go service. Cloud computing is a shared pool of configurable computing resources. According to the National Institute of Standards and Technology (NIST) definition, "cloud computing is a model for enabling ubiquitous, convenient, on-demand network access to a shared pool of configurable computing resources (e.g., networks, servers, storage, applications, and services) that can be rapidly provisioned and released with minimal management effort or service provider interaction." A cloud computing architecture can be deployed using public, private or hybrid models [4]. Many organizations or institutes prefer private cloud as security is a concern. A private cloud is hosted within the organization, behind the firewall, intended to use by an organization itself and no external entities should get access to it [5]. In such cases, the organization invests in and manages its cloud infrastructure, but gains benefits from pooling a smaller number of centrally maintained high-performance computing and storage resources instead of deploying large numbers of lower performance systems. Cloud computing services are divided into four deployment models, and three cloud computing services discussed below [1].

\subsection{Cloud Service Model:}

1.1.1 Software as a service (SaaS) allows accessing software online over the Internet on cloud platform applications. E.g. Office 365, Google Apps, Netflix.

1.1.2 Platform as a service (PaaS) delivers hosted development sets, and application management capabilities. One can code, deploy, manage applications and launch software application. E.g. Google App Engine, Microsoft Azure.

1.1.3 Infrastructure as a service (IaaS) is the most efficient and basic service model in cloud computing which provides physical and virtual resources. E.g. Amazon Web Services (AWS).

\subsection{Cloud computing four deployments models:}

\subsubsection{Private Cloud}

According to organizations rules and policies, the deployment of private cloud is carried out for on-premises. It is mostly dedicated to a single organization. Multi-tenant and Scalability are some features of this model. E.g. Rackspace and VMware companies provide private cloud facility.

\subsubsection{Public Cloud}

Public Cloud shares all its resources among the public. The public cloud deployment model is used where security or confidentiality of information is not an issue. Third parties offering the services are involved in the public cloud. The public cloud deployment reduces the costs and increases the 
use of technology. Using Google is one of the example.

\subsubsection{Hybrid Cloud}

Hybrid cloud is combined usage of private and public cloud as required. One unique aspect of the hybrid cloud is that it could be managed by more than one service providers as compared to other models. The hybrid architecture provides better deployment opportunities to organizations. It is helpful for the organizations where a change of cloud environment is constant.

The paper discusses Cloud Computing in Education in Section II, and Observations in Section III, followed by Conclusion in Section IV.

\section{CLOUD COMPUTING IN EDUCATION}

\subsection{Education System Model}

The proposed Education System Model Using Cloud Computing designed for school age group students explains the connectivity and sharing of data among teachers, students, staff and parents/guardians as well. It provides an idea of how cloud computing can be used in education to overcome the challenges faced. The paper proposes Parents/Guardian Login functionality be added in the education system model making it easier for parents to keep updates of activity records and grades of their respective child [1].

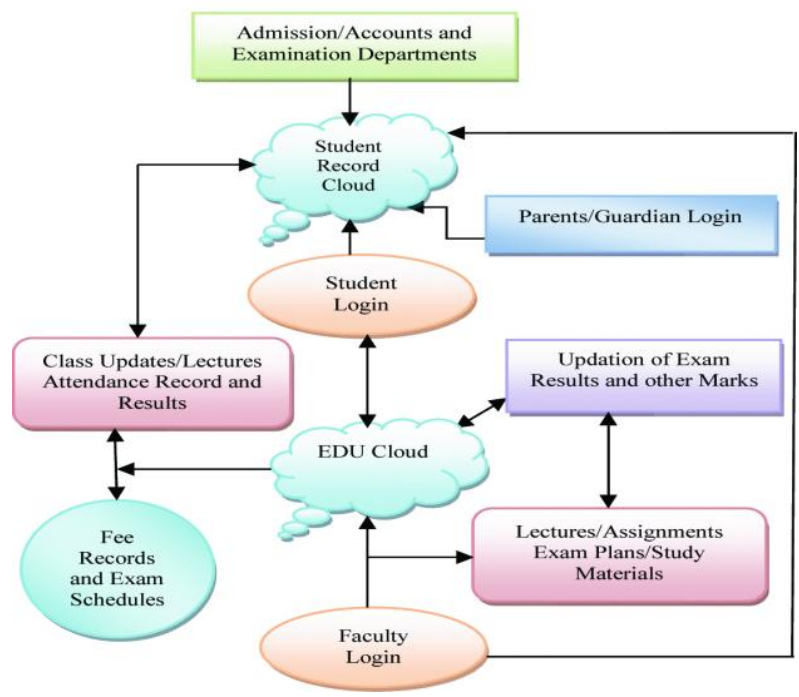

Fig 1: Education System Model Using Cloud Computing [1]

\subsection{Cloud Wisdom Education Architecture}

It is a commercial platform based on Service-oriented Architecture (SOA) framework theory and cloud computing that solves the issues regarding information island and resources utilization [2]. The purpose of SOA technology is to solve Information Island, realize data resource sharing and cooperation with all departments, achieving quick response in digital educational development. The computing framework of high-performance cloud wisdom education platform divided into three layers known as IaaS, PaaS, and SaaS [2][3]. By integrating SOA and cloud computing, the cloud wisdom education platform is designed. It constructs the three-layer application framework of data resources that has Application layer, Data service layer, and Storage layer which solves problems like the island of educational teaching resources, scattered educational teaching resources, and low data handling capacity. The three layers are Application layer, Data service layer, and Storage layer. The Integration SOA and cloud resource sharing improve the hardware utilization rate because without SOA there is no cloud computing. To solve data isolation of multiple, the author proposed a tenant management method under multi-tenant mode. As the platform is registered, it solves the problem of user propriety. Tender_ID refers to the ID of tenants. User id means ID of users. This method serves customized services for service providers and isolates data between two tenants to ensure completeness and confidentiality of tenant's data [2].

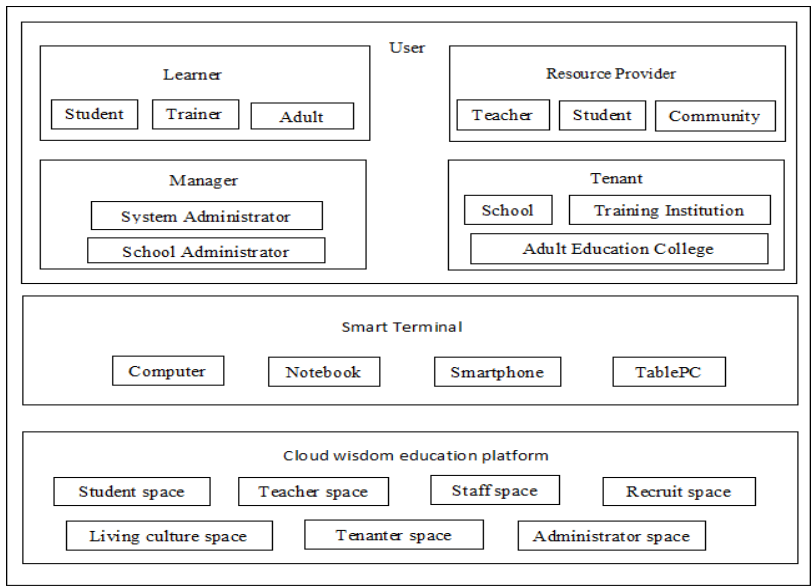

Fig 2: Cloud Computing Framework of SaaS [2]

\subsection{V.I.C.E}

Vertical Architecture termed as V.I.C.E stands for Vertical Implementation of a Cloud for Education. The important feature of the architecture is simplicity and scalability. The best suitable deployment model chosen for V.I.C.E architecture is Infrastructure as a Service (IaaS) that allow users to access computing and storage resources on demand. Hence proposed architecture is vertical IaaS cloud known as V.I.C.E. The only goal is the cost-effective approach of cloud lab than traditional computer labs. The experimental method was to use $50 \mathrm{RPi}$, low-cost tiny computers instead of 50 Dell devices; it achieves the cost-effective approach. V.I.C.E architecture is made of six machines from which five machines are computed nodes, and one is the head node. The five compute nodes are termed as com1, com2, com3, com4, com5. Com1, com2, and com3 comprises of ECS BAT-I (1.2) J1800 Intel Celeron J1800 $2.41 \mathrm{GHz}$ Mini ITX Motherboard/CPU/VGA Combo. Com2 and com3 each possess 4GB RAM. Com1 has an additional 4GBs of RAM and a 64GB SATA III MLC Internal Solid State Drive (SSD). The three compute nodes, i.e., com1, com2, and com3 each can host at least 6 Virtual Machines (VMs). Com4 and com5 have Intel Core i5 quad-core processors, 128GB SATA III Internal SSD, and 16GB RAM. It allows them to support at least $16 \mathrm{VMs}$ each. The head node has an Intel Core i3 DualCore processor and 8GB of RAM. It serves as the point in which scheduling and load balancing are handled. It serves as home for the load balancer, hosts web interface using which users connect to architecture to launch VMs. They implemented their own middleware coded in a python programming language to manage V.I.C.E architecture [3].

\subsection{Private Cloud using OpenStack}

Higher education in Information Technology lab course may require specific softwares of specific operating system compatible with particular hardware machine. Need for different operating systems requirement, installation, and 
maintenance process poses a difficulty to manage them. OpenStack addresses this issue by providing IaaS for hardware resources and a standard mechanism to manage and distribute course specific VMs. The paper presents a private cloud for higher education and proof-of-concept implementation methodology for the OpenStack platform. It presents an implementation and design of private cloud prototype using OpenStack. The major goal of OpenStack is scalability. OpenStack platform because it enables students and researchers to work with ready to use pre-configured virtual machines depending on the course requirement.

The deployment method states installation parameters and resource requirement model. Under installation parameters, OpenStack based private cloud is deployed on two commodity servers. KVM hypervisor and RPM distribution of OpenStack (RDO) is selected and deployed on top of CentOS. RDO is a freely-available, community supported Red Hat's distribution of OpenStack. The Packstack installation tool used is a command-line based installation tool specific to the RDO. Packstack enables rapid deployment of OpenStack on existing servers. The services selected are Dashboard, Compute, Identity, Networking, Image, Block Storage and Object Storage installed and configured by an administrator on a cloud as required [4].

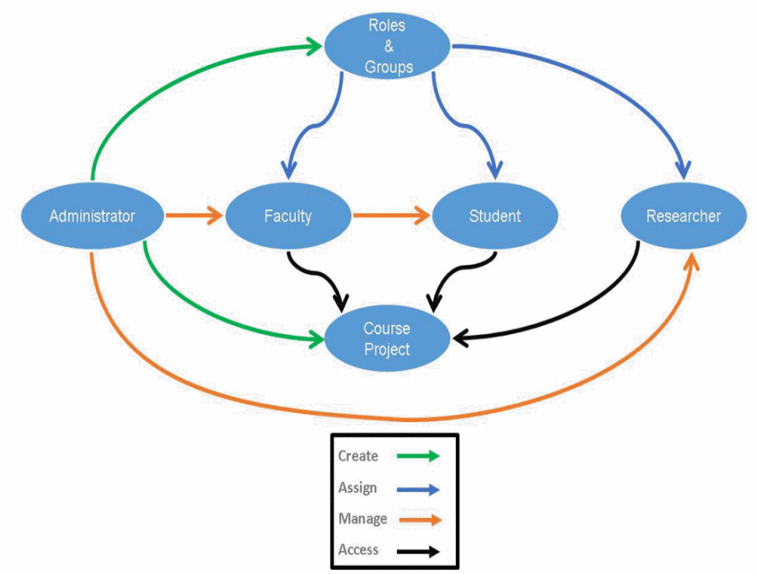

Fig 3: HEI Private Cloud Design [4]

\subsection{Selecting the platform for MECA- Cloud}

MECA (MicroElectronics Cloud Alliance) BME aims at building an educational cloud and host one active node of a Europe wide cloud platform to support the education at the field of microelectronics. The system architecture and realization process for open education of microelectronics is done. The approach used for building educational cloud is IaaS as it provides us bare infrastructure of resources like computational and storage capacity, operational memory, network bandwidth, etc. according to demands and usage.

A Kernel-based Virtual machine (KVM) hypervisor is used in IaaS as a hosted technology for the guest system [8]. For high performance and scalability, the basic installation of Apache CloudStack is required at each participant. The installation requires the creation of one zone containing one pod containing one cluster containing one or more hosts was preferred. Three physical machines are applied in the cloud built by the department. The computational node is HP server. A PC used as a firewall provisioning pfSense, and another PC hosts two VMs using KVM. For high-speed intranet network, gigabit switch is used. Apache Cloudstack installation offers a simple integration of basic management data of the CloudStack Zones, i.e., the nodes of the partners. The partners must install their CloudStack using a strict distribution of the region IDs. The three partners participating in the pilot project assigned region IDs as follows: Polito got region ID 1, eWorks region ID 2 and BME ID 3 respectively. The actual migration of the user data is realized by simple SQL commands in a well-specified running order and logic. A private cloud platform has been set up at three project partners participating in the proof of concept pilot for building the MECA cloud. The Cloudstack from Apache is selected for the realization. Starting from a single installation, all three partners (Polito, eWorks, and BME) created a private cloud with one Zone, one Pod and one Cluster at their site [5].

\section{6 eduDScloud}

eduDScloud (educational data analytics and service-oriented architecture cloud) architecture only goal is to give the students hands-on experience with distributed big data processing, data storage and data mining in the data lab. eduDScloud prevents a vendor lock-in issue of the cloud. Vendor lock-in does not allow customers to choose or opt for another service or a product while using the current service, i.e., it prevents the customer from switching to another service provider or vendor of that product or service. It is also called as customer lock-in or proprietary lock-in. Vendor lock-in settled by avoiding dependencies on platform-specific features where possible, like the concept and significant parts of lab setups and especially virtual machine themselves were made portable enough to switch between different vendors [6].

eduDScloud is implemented based on VMware vCloud Director (vCD) and accompanying products like ESXi (Hypervisor) and vSphere due to pre-existing infrastructure. vCD presents the lab in the form of vApp. This vApp is displayed as an instance from the templates stored in the catalog. One instance is used in every group of vApp and one instance in the common services vApp. The idea is to give every group its own distributed file system for experiments. To store data, Apache Hadoop and contained an HDFS file system is used. $\mathrm{vCD}$ and pfSense are authentication providers. pfSense is a gateway that allows students to connect to an internal network and gain internet access. pfSense acts as an OpenVPN server and gateway. vCD uses permission system to manage authorization, and these permissions are synced to a respective pfSense instance. Apache Spark is used as an alternative to Hadoop that supports in-memory processing of data stored distributed over the RAM of all cluster nodes. Having both frameworks installed allow teaching the students these issues. For both Hadoop and Spark, YARN resource manager is used. eduDScloud provides a scalable private cloud solution for hosting virtual labs. It allows students to have hands-on experience with root access to virtual machines and network within a sandboxed environment. Also, the architecture enables lecturers to devise complex tasks with minimum administrative work because new labs are instantiated using pre-built templates [7].

\subsection{VPPL}

Virtual Programming Practice Lab (VPPL) architecture extends the capability of computer labs to use existing workstations of a computer lab for programming courses offered at Kabul Polytechnic University (KPU) because the number of students exceeds the number of resources. The solution proposed is to use oVirt an open source virtualization platform to manage virtual computers for the users and to configure servers and install equipment which is required for use in programming lab activities. The architecture was 
proposed because students were not satisfied by the unavailability of labs and laboratories equipment. Therefore system grant students to have their individual virtual computer in an academic semester, this will facilitate students to save their assignments or tasks for the next time use of computer and also, they will be able to access their virtual PC at any time remotely.

For operating Virtual PC, Users are asked to install Virt Viewer tool like virtual box on their PC, a tool that displays the graphical console of the VM. The main back-end components of the system are nodes and engine. The System is divided into two parts, Private Network, and Public Network. The oVirt engine is control center of the VPPL architecture that allows defining host, networking and creating VM and also configuring data center and cluster. oVirt nodes are Type 1 kernel based VM hypervisor created by the ovirtnode team. Nodes are located on a private network and can be added or removed by the engine; every node can host multiple VMs depending on their hardware capabilities. VDSM a daemon required by a Virtualization Manager such as oVirtengine is used to manage the KVM guests in a lab environment. The oVirt engine operates on CentOS operating system in a separate computer [8].

\subsection{Information Security for Cloud}

Cloud in the education sector plays an important role nowadays. With the widespread use of cloud in the education sector there arises the need for security as well. The Education Cloud Platform provided service for teachers, students, and parents in various education departments and schools in Shenzhen. The design architecture of educational cloud computing platform based on the CentOS system that connects to the network through the Huawei S9706 and the Huawei USG5530 boundary firewall connected to the telecommunications Internet. Using the tool of LoadRunner and AppScan, the Design architecture of the educational cloud computing platform could be tested, analyzed. Analysis of the information security is carried out for the Education cloud platform system in Longgang District, Shenzhen City. After examining risks in the cloud, the next step is to mitigate those risks and modify high-security risk issues in education cloud platform or application system. Mitigating risks will improve the personal privacy issues of the education department, teachers and students thereby advancing the quality of teaching. The paper only focuses on the security perspective of the education cloud platform. The tools used are:

\subsubsection{LoadRunner}

A software testing tool used to test applications, measuring system behavior, and performance under load.

\subsubsection{AppScan}

A web security testing and monitoring tools from the Rational Software division of IBM. It is intended to test Web applications for security vulnerabilities during the development process when it is least expensive to fix such problems.

The steps of AppScaning experiment are:

a. Verify if there is a security hole by analyzing a response

b. AppScan scanning principle $=$ scanning rules + crawling + testing.

\section{OBSERVATION}

\subsection{Cloud Computing vs. Traditional Computing}

The private cloud computing model is used for security purpose and presentation of cloud virtual labs instance for better understanding of cloud that saves time and money by investing in traditional IT infrastructure. Using resources virtually is a cost-effective approach, and beneficial to save cost and space consumed by IT infrastructure, as data is either hosted within the organization, at a data center or by a trusted third party. Refer Table 1 [10] and Table 2.

Table 1: Traditional Computing vs. Cloud Computing

\begin{tabular}{|c|c|c|}
\hline Benefits & $\begin{array}{c}\text { Traditional } \\
\text { Computing }\end{array}$ & Cloud Computing \\
\hline Pricing & $\begin{array}{c}\text { Expensive and large } \\
\text { capital cost for IT } \\
\text { infrastructure } \\
\text { implementation. } \\
\text { e.g. 50 Dell 745s } \\
24,000 \text { (In USD) [3]. }\end{array}$ & $\begin{array}{c}\text { Economical and } \\
\text { feasible, pay as you } \\
\text { service payment for } \\
\text { the resources used. } \\
\text { e.g. 50 Raspberry } \\
\text { Pis for 4,000 (In } \\
\text { USD) [3]. }\end{array}$ \\
\hline Infrastructure & $\begin{array}{c}\text { Costly standalone } \\
\text { server hardware and } \\
\text { server software }\end{array}$ & $\begin{array}{c}\text { Multi-tenant } \\
\text { infrastructure } \\
\text { managed by vendor }\end{array}$ \\
\hline Maintenance & $\begin{array}{c}\text { Purchased from the } \\
\text { vendor and deployed } \\
\text { by the client to } \\
\text { affected local servers } \\
\text { and clients. }\end{array}$ & $\begin{array}{c}\text { Included in } \\
\text { subscription fee } \\
\text { and managed } \\
\text { entirely by vendor. }\end{array}$ \\
\hline Scalability & $\begin{array}{c}\text { Increase in usage } \\
\text { requires costly } \\
\text { hardware upgrades }\end{array}$ & $\begin{array}{c}\text { Add users or } \\
\text { increase } \\
\text { transactions at any } \\
\text { time }\end{array}$ \\
\hline
\end{tabular}

Table 2: Observations Table

\begin{tabular}{|c|c|c|}
\hline & Related Work & Observations \\
\hline 1. & $\begin{array}{c}\text { Education System } \\
\text { Model }\end{array}$ & $\begin{array}{l}\text { Cloud system designed for } \\
\text { school age group. Reliable } \\
\text { for students to get updated } \\
\text { notes and login } \\
\text { functionality for } \\
\text { parents/guardian to keep a } \\
\text { track of students activities. }\end{array}$ \\
\hline 2. & $\begin{array}{c}\text { Cloud Wisdom } \\
\text { Education Architecture }\end{array}$ & $\begin{array}{l}\text { To realize the issues } \\
\text { regarding information } \\
\text { island and low teaching } \\
\text { resource utilization. A } \\
\text { framework designed for } \\
\text { each service model } \\
\text { respectively i.e. IaaS, PaaS } \\
\text { and SaaS. }\end{array}$ \\
\hline 3. & $\begin{array}{c}\text { V.I.C.E } \\
\text { (Vertical } \\
\text { Implementation of } \\
\text { Cloud for Education) }\end{array}$ & $\begin{array}{l}\text { Cost effective model or } \\
\text { approach termed as } \\
\text { V.I.C.E that replaces the } \\
\text { traditional IT } \\
\text { infrastructure with low } \\
\text { cost tiny computers (i.e. } \\
\text { Raspberry Pis) for virtual } \\
\text { cloud labs. }\end{array}$ \\
\hline
\end{tabular}




\begin{tabular}{|c|c|c|}
\hline 4. & $\begin{array}{c}\text { Private cloud } \\
\text { implementation using } \\
\text { OpenStack }\end{array}$ & $\begin{array}{l}\text { To offer services like } \\
\text { installation and running of } \\
\text { different software's } \\
\text { running on different } \\
\text { Operating Systems i.e. an } \\
\text { alternative method to } \\
\text { comprise many different } \\
\text { machines in lab. Cost and } \\
\text { energy efficient approach } \\
\text { as an alternative for } \\
\text { traditional IT labs [4]. }\end{array}$ \\
\hline 5. & $\begin{array}{l}\text { Selecting the platform } \\
\text { for MECA-Cloud }\end{array}$ & $\begin{array}{l}\text { Private cloud setup for } \\
\text { three project partners } \\
\text { (Polito, eWorks, and } \\
\text { BME). It focuses on } \\
\text { choosing the right } \\
\text { approach i.e. choosing the } \\
\text { right platform and } \\
\text { configuration. Virtual } \\
\text { machines with software } \\
\text { tools must be created to } \\
\text { support Microelectronics } \\
\text { field of study [5]. }\end{array}$ \\
\hline 6. & $\begin{array}{c}\text { eduDScloud } \\
\text { (educational data } \\
\text { analytics and service- } \\
\text { oriented architecture } \\
\text { cloud) }\end{array}$ & $\begin{array}{l}\text { Provides private cloud } \\
\text { infrastructure and } \\
\text { sandboxed environment } \\
\text { for students to carry out } \\
\text { data analytics lab } \\
\text { experience granting } \\
\text { students with full access to } \\
\text { machines and their own } \\
\text { internal network. }\end{array}$ \\
\hline 7. & $\begin{array}{l}\text { Virtual Programming } \\
\text { Practice Lab (VPPL) }\end{array}$ & $\begin{array}{l}\text { VPPL is intended to } \\
\text { simulate the environment } \\
\text { for students to perform } \\
\text { programming activities. } \\
\text { oVirt solution is proposed } \\
\text { to install equipments } \\
\text { needed, manage servers } \\
\text { and virtual computing } \\
\text { resources important for lab } \\
\text { activities. }\end{array}$ \\
\hline 8. & $\begin{array}{l}\text { Information Security for } \\
\text { Cloud }\end{array}$ & $\begin{array}{l}\text { The security for cloud } \\
\text { platforms activity is } \\
\text { proposed by using two } \\
\text { such tools those are } \\
\text { LoadRunner and AppScan. } \\
\text { These applications are } \\
\text { used to test and analyze } \\
\text { the security of the cloud } \\
\text { platforms. }\end{array}$ \\
\hline
\end{tabular}

\section{CONCLUSION}

Cloud computing is an advanced development in the modern technology era. Currently, it is an emerging discipline that helps the IT and Business industries to get efficient use of their Hardware and Software resources and enabling serviceoriented, on-demand network access to rapidly scalable resources with promises to cut operational and capital cost. It has a broad aspect of computing application and provides a global platform for people. It is helpful for every sector from enterprise users to an educational purpose. Apart from traditional education, Students will have the benefit of online learning provided through the internet or cloud. Institutes of weak financial state can have high computing facilities within low cost. The paper discussed the multiple implementations of the cloud platform for the education sector creating virtual labs using available services like OpenStack or code for their own experience. The suitable deployment model selected was IaaS as it gives benefit to install whatever applications desired and security measures related to it. Building a Private Cloud ensures that the connection is secure and safe from the outside world and needs authentication and authorization factor for the external network to connect to private clouds internal network.

\section{ACKNOWLEDGMENT}

Research reported in this publication was supported by K J Somaiya College of Engineering, Vidyavihar Mumbai, University of Mumbai. Special thanks to Benu George (Ph.D. student, Research Scholar, NIT Calicut) for guidance and support.

\section{REFERENCES}

[1] E. A. Ahmed and H. Ali Ahmed, "A Proposed Model for Education System Using Cloud Computing," in 2018 3rd International Conference on Emerging Trends in Engineering, Sciences and Technology (ICEEST), 2019, pp. 1-4.

[2] H. Jingzhao, "Research on the wisdom education platform of cloud computing architecture," 3rd IEEE Int. Conf. , pp. 1-5, 2017.

[3] T. Brummett and M. Galloway, "Building a Vertical Cloud Architecture for Education," IEEE Int. Conf. Cloud Comput. CLOUD, vol. 2018-July, pp. 540-547, 2018.

[4] G. Bhatia, I. Al Noutaki, S. Al Ruzeiqi, and J. Al Maskari, "Design and implementation of private cloud for higher education using OpenStack," Proc. Majan Int. Conf. Promot. Entrep. Technol. Ski. Natl. Needs, Glob. Trends, MIC 2018, pp. 1-6, 2018.

[5] P. Martinek et al., "Building a cloud platform for education in microelectronics," Proc. Int. Spring Semin. Electron. Technol., pp. 1-6, 2017.

[6] J. Opara-Martins, R. Sahandi, and F. Tian, "Critical analysis of vendor lock-in and its impact on cloud computing migration: a business perspective," J. Cloud Comput., 2016.

[7] A. Koschel, F. Heine, I. Astrova, and I. Astrov, "A private cloud for data mining education," Proc. - 2018 6th Int. Conf. Enterp. Syst. ES 2018, pp. 129-133, 2018.

[8] M. A. Sultani, M. N. Kabiri, and M. Wannous, "Utilization of cloud technologies in building a virtual programming lab for higher education in Afghanistan work in progress," Proc. 4th IEEE Int. Conf. Appl. Syst. Innov. 2018, ICASI 2018, pp. 422-425, 2018.

[9] W. Nie, X. Xiao, Z. Wu, Y. Wu, F. Shen, and X. Luo, "The research of information security for the education cloud platform based on appscan technology," Proc. - 5th IEEE Int. Conf. Cyber Secur. Cloud Comput. 4th IEEE Int. Conf. Edge Comput. Scalable Cloud, CSCloud/EdgeCom 2018, pp. 185-189, 2018.

[10] Rackspace, “Advantages of Cloud Computing," Racksp. Us, Inc., no. 519, p. 3100, 2016. 